\title{
eJRIEPS
}

Ejournal de la recherche sur l'intervention en éducation physique et sport

$41 \mid 2017$

Varia

\section{Les conflits vécus par le tuteur ESPE et le tuteur EPLE dans le dispositif du tutorat mixte}

\section{Sylvie Moussay, Véronique Blanjoie et Patrick Babut}

\section{(2) OpenEdition \\ Journals}

Édition électronique

URL : http://journals.openedition.org/ejrieps/699

DOI : 10.4000/ejrieps.699

ISSN : 2105-0821

Éditeur

ELLIADD

Référence électronique

Sylvie Moussay, Véronique Blanjoie et Patrick Babut, «Les conflits vécus par le tuteur ESPE et le tuteur EPLE dans le dispositif du tutorat mixte », eJRIEPS [En ligne], 41 | 2017, mis en ligne le 01 avril 2017, consulté le 01 août 2019. URL : http://journals.openedition.org/ejrieps/699 ; DOI : 10.4000/ ejrieps.699

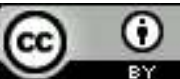

La revue eJRIEPS est mise à disposition selon les termes de la Creative Commons Attribution 4.0 International License. 


\section{eJRIEPS 41 avril 2017}

\section{Les conflits vécus par le tuteur ESPE et le tuteur EPLE dans le dispositif du tutorat mixte}

Sylvie Moussay, Véronique Blanjoie et Patrick Babut

ESPE Université Clermont-Auvergne, Laboratoire ACTé, France

\section{Résumé}

Cet article présente une étude sur le travail réel des tuteurs impliqués dans le tutorat mixte d'enseignants stagiaires en éducation physique et sportive. Le tutorat mixte associant un tuteur ESPE (École supérieure du professorat et de l'éducation) et un tuteur EPLE (Établissement public local d'enseignement) fait partie des nouveaux dispositifs dont l'objectif est de renforcer l'accompagnement des stagiaires à l'appui d'un collectif de formation. Inscrite dans l'approche clinique de l'activité (Clot, 2008), l'étude analyse les dimensions contrariées de l'activité vécues par les deux tuteurs au cours de la visite formative. A partir de l'analyse des données d'entretiens d'autoconfrontation simple et des données de confrontation collective, les résultats permettent d'apprécier l'intérêt $d u$ dialogue collectif comme lieu d'émergence et de formalisation des conflits d'activité individuelle et collective. Des pistes de formation sont proposées dans la perspective d'aider les tuteurs ESPE et les tuteurs EPLE à développer des ressources pour travailler ensemble.

Mots-clés : tutorat mixte, analyse de l'activité, conflit, collectif de formation, clinique de l'activité

\section{Introduction}

En réaffirmant en 2013 le principe d'alternance intégrative, la réforme sur la refondation de l'école de la République (loi 2013-595, 8 juillet 2013) accorde une nouvelle dimension au dispositif d'accompagnement des futurs enseignants. Les perspectives d'un tutorat mixte associant un tuteur ESPE (École supérieure du professorat et de l'éducation) et un tuteur EPLE (Établissement public local d'enseignement) confirment la nécessité de renforcer le partenariat entre les universités et les établissements scolaires d'affectation des stagiaires (Coffey, 2010 ; Gervais, 2008). Selon la littérature scientifique du domaine, l'évolution de l'accompagnement des stagiaires vers un tutorat fondé sur une dynamique collaborative 


\section{eJRIEPS 41 avril 2017}

(Van Nieuwenhoven, Colognesi, Wattiez \& Bothy, 2016 ; Wang, \& Ha, 2012) et une pratique concertée entre les formateurs permet aux stagiaires de construire plus facilement des liens entre les savoirs théoriques et pratiques (Boutet \& Pharand, 2008). Sur ce point, les textes de cadrage des mises en stage professionnel proposés par les ESPE affirment la plus-value du tutorat mixte, celui-ci étant envisagé comme un dispositif ouvert sur un décloisonnement des lieux d'apprentissage en vue de favoriser le continuum du processus de formation des enseignants. Plus particulièrement, dans le champ de l'intervention en éducation physique et sportive, certaines propositions innovantes démontrent l'impact réel d'un tutorat collaboratif sur le développement professionnel des stagiaires (Chaliès, Bruno-Méard, Méard \& Bertone, 2010 ; Chaliès, Escalié \& Bertone, 2012 ; Ciavaldini-Cartaut, 2012 ; Meegan, Dunning, Belton, \& Woods, 2013). Pour l'essentiel, ces propositions montrent l'intérêt pour les deux tuteurs d'opérer une sélection concertée des événements marquants de la séance du stagiaire (Chambers \& Armour, 2012) pour s'engager dans une activité collective de confrontation et de mise en adéquation des interprétations sur l'expérience du stagiaire (Escalié \& Chaliès, 2009). Elles soulignent également les circonstances dans lesquelles une analyse conjointe des formateurs sur l'activité et un double étayage favorisent chez le stagiaire une démarche réflexive sur son travail. Les entretiens de conseil menés par les deux tuteurs sont ainsi envisagés efficaces lorsqu'un partenariat réflexif et collaboratif est établi (Bertone \& Saujat, 2013). Dans ces études, l'utilité du travail collaboratif du tuteur ESPE et du tuteur EPLE et la façon de « s'y prendre à plusieurs » est mise en avant, notamment lorsque ces deux professionnels accompagnent les stagiaires à transformer leur expérience d'enseignement au moyen de leur interprétation avec les règles et les concepts professionnels de la formation (Bertone, Chaliès, \& Clot, 2009). A travers d'autres études, certains dispositifs de tutorat relevant de la vidéoformation et d'une démarche d'analyse de l'activité apparaissent novateurs (Ciavaldi-Cartaut, 2016). Les résultats rendent compte de la façon dont les deux tuteurs s'appuient sur les traces audiovisuelles de l'activité pour aider le stagiaire à développer ses actions et ses motifs d'agir. Sur ce point, l'aménagement du tutorat fondé sur l'usage d'outils numériques s'inscrit dans le cadre évolutif de la formation en France des enseignants d'EPS (Flandin \& Ria, 2014 ; Roche \& Gal-Petitfaux, 2014). Certains modules d'enseignement orientés « activité » accordent une place importante aux outils réflexifs et méthodologiques pour guider l'observation des étudiants et l'analyse des pratiques en éducation physique et sportive (Gaudin, Perrot, Chaliès, Escalié \& Raymond, 2014). 


\section{eJRIEPS 41 avril 2017}

Cependant, si les expérimentations locales de tutorat collectif et les résultats des travaux d'étude convergent sur un certain nombre de principes (construction d'une activité conjointe des tuteurs, complémentarité des regards sur le travail du stagiaire), il semble que le tutorat mixte se réduit encore à une collection d'individus exposés à l'isolement (...) chacun individuellement se trouve alors confronté aux mauvaises surprises d'une organisation du travail qui laisse «sans voix » face au réel (Clot, 2005, p.190). En ce sens, l'inconsistance du collectif «Tuteur ESPE- Tuteur EPLE » est une nouvelle fois soulignée même si le dispositif du tutorat mixte facilite la rencontre entre les différents formateurs (Moussay \& Serres, 2015). Un rapport de l'Inspection Générale (IGAENR, 2015) précise notamment les difficultés de la mise en place du tutorat mixte. II souligne que la contribution de chaque tuteur reste imprécise, parfois problématique, et les concertations au sein de la triade encore insuffisantes et peu structurées collectivement. Ce qui rejoint en partie les résultats de recherche mettant en avant la complexité des échanges au sein d'une triade où les acteurs ont des préoccupations différentes.

La perspective d'une alternance intégrative effective (Rapport IGEN-IGAENR, 2015) à travers le tutorat mixte est interrogée tout comme l'écart entre l'intention visée par l'institution et la réalité du travail du tuteur ESPE et du tuteur EPLE. Par ailleurs, certains travaux notent un paradoxe entre une sur-prescription d'objectifs sur le tutorat collaboratif et une sous-prescription de moyens qui confronte parfois les deux tuteurs à des difficultés professionnelles leur imposant de «prendre sur eux» pour concevoir des modalités de travail inédit. L'ensemble de ces considérations invite à poursuivre l'étude du travail réel du tuteur ESPE et du tuteur EPLE et à analyser précisément les conflits vécus par ces professionnels lorsqu'ils sont amenés à travailler ensemble au cours de la visite formative. Dans ce texte, la question du conflit est abordée selon une perspective clinique de l'activité (Clot, 2008). Suite à la description du cadre conceptuel de l'étude, le choix des différentes étapes méthodologiques est justifié, notamment le protocole organisant la confrontation des tuteurs aux traces audiovisuelles de leur activité et engageant ces professionnels dans un travail de formalisation des conflits de l'activité. Les résultats de l'étude permettent d'apprécier la façon dont les tuteurs construisent et développent la signification des conflits de l'activité collective. Enfin, à l'appui des résultats, la discussion est organisée autour de quelques pistes de réflexion sur la formation professionnelle des tuteurs ESPE et des tuteurs EPLE afin d'optimiser le travail collectif. 


\section{eJRIEPS 41 avril 2017}

\section{Cadre théorique de l'étude}

Notre étude repose sur le cadre conceptuel de la Clinique de l'activité en Psychologie du travail. En référence à cette approche, l'activité ne peut pas uniquement être appréhendée à partir de la tâche prescrite ; elle prend en compte l'activité réalisée et le réel de l'activité c'est à dire ce que le sujet a voulu faire et qu'il n'a pas pu faire, ce qu'il n'a pas renoncé à faire sans pour autant y parvenir, ce qu'il a fait pour ne pas faire ce qui était à faire (Clot, 2008, p. 89). L'activité comprend les « activités suspendues, contrariées ou empêchées, voire les contre-activités qui doivent être admises dans l'analyse du travail » (Clot, 1999, p.120). Ces activités suspendues, contrariées, empêchées sont l'expression de conflits intrapsychiques auxquels est confronté tout professionnel. Les conflits sont ainsi envisagés comme une caractéristique propre à l'activité psychique humaine. En référence à certains travaux dans le champ de la formation des enseignants (Bertone, Méard, Ria, Euzet, Durand, 2003), le conflit intrapsychique (Vygotski, 1934/1997) désigne le processus selon lequel l'acteur évoque des actions concurrentes, possibles à réaliser au regard de la situation présente qu'il évalue. Le choix d'une action comporte l'inhibition de certaines actions possibles au profit d'autres qui s'imposent dans la situation présente. Selon Bertone ${ }^{1}$, le dilemme est un cas particulier du conflit et correspond aux situations où l'acteur doit décider d'actions à réaliser qu'il considère lui-même comme contradictoires ou incompatibles. Dans l'étude présentée, le postulat consiste à envisager le tutorat mixte comme une situation de travail pouvant générer des conflits du fait de la concurrence des actions (but) et des motifs (ce qui oriente l'activité) énoncés par chaque tuteur à propos du travail collectif.

Dans une perspective développementale, les conflits peuvent être une source de développement pour transformer la situation de travail ou au contraire un obstacle s'ils ne parviennent pas à être réinvestis dans le travail (Quillerou-Grivot \& Clot, 2013). Le dialogue collectif apparaît comme une voie féconde de développement des conflits sur la base de compromis et de négociation d'actions alternatives. II s'agit d'organiser le dialogue entre les professionnels et les conflits mais aussi entre eux sur ces conflits (Balas, 2011). Dans cette perspective, l'étude présentée s'est positionnée dans le paradigme du «transformer pour comprendre » (Clot, 2008) en vue de provoquer le développement de la signification du conflit. En ce sens, les conditions d'un redoublement de l'expérience, « d'une expérience au carré », par le dialogue collectif peuvent être

\footnotetext{
${ }^{1}$ Texte «L'activité humaine entre migration et conflit intra-psychique » rédigé par Stefano Bertone à partir de la présentation d'Yves Clot du 7 janvier 2005 au CNAM Paris
} 


\section{eJRIEPS 41 avril 2017}

l'occasion pour les professionnels de développer une nouvelle formalisation des conflits générés par la situation de travail. Dans ces conditions, les conflits peuvent devenir des éléments caractéristiques du métier et constitutifs du patrimoine générique (Clot \& Faïta, 2000) en se parant d'une dimension transpersonnelle (Clot, 2008).

\section{Méthode}

\section{1. Le contexte de l'étude}

L'étude a été réalisée au cours de l'année universitaire 2015-16 en collaboration avec les tuteurs de l'académie de Clermont-Ferrand. Elle s'est appuyée sur un constat général d'incompréhension exprimé par certains tuteurs EPLE à l'égard du tutorat mixte. En effet, lors d'une journée de formation, les tuteurs EPLE ont évoqué la difficulté à planifier des temps de travail collectif avec les tuteurs ESPE, difficulté due principalement à des contraintes d'emploi du temps. Ils ont exprimé « l'inenvisageable » c'est à dire les activités impossibles à réaliser parce qu'elles n'étaient pas autorisées par les textes de cadrage à l'accompagnement des fonctionnaires stagiaires. C'était le cas par exemple des fiches de positionnement que les tuteurs EPLE devaient renseigner, transmettre au Rectorat sans autorisation de le partager avec les tuteurs ESPE. Enfin, les tuteurs EPLE ont mentionné l'absence de formation commune avec les tuteurs ESPE, soulignant ainsi le contexte peu propice à une organisation collective de la visite formative.

Suite à ce constat, nous avons sollicité deux binômes (tuteur/rice ESPE et tuteur/trice EPLE) en leur expliquant tout d'abord l'intention de mener une étude sur les dimensions contrariées de leur travail collectif. Notre sollicitation s'est accompagnée d'échanges autour des travaux sur le tutorat dans le champ de l'Éducation physique et sportive, particulièrement les études ayant formalisé, à partir des concepts de conflits et de dilemmes, les difficultés vécues par le tuteur lors des entretiens post-leçons (Chaliès, Cartaut, Escalié \& Durand, 2009). Afin d'acculturer les tuteurs au cadre conceptuel de l'étude, nous avons également défini le tutorat mixte comme une situation de travail collectif au sens de «travailler ensemble, coopérer, collaborer et s'entraider » (Cuvelier \& Caroly, 2009, p.61) et échangé sur les enjeux d'un collectif de formation dans le cadre de l'accompagnement professionnel des stagiaires (Escalié \& Chaliès; 2012 ; Moussay, 2012). Enfin, nous avons précisé les modalités de participation (enregistrement filmique du travail suivi d'un entretien d'autoconfrontation simple puis d'une confrontation collective). Deux précautions ont été mises en avant concernant les données d'enregistrement: obtenir de la part de chaque élève une autorisation parentale pour garantir le droit à 


\section{eJRIEPS 41 avril 2017}

l'image et avoir l'accord des tuteurs et des stagiaires pour exploiter les matériaux recueillis dans des projets de communication et de publication scientifiques.

\section{2. Les participants}

Deux binômes de tuteurs/trices en éducation physique et sportive (un binôme étant constitué d'un tuteur ESPE et d'un tuteur EPLE) ont participé à cette étude. Afin de garantir toute confidentialité, les prénoms des participants ont été modifiés.

Binôme 1 : Anna, enseignante en éducation physique et sportive (EPS), âgée de 47 ans, assurait pour la première fois la fonction de tutrice en établissement d'un professeur stagiaire $^{2}$, sans avoir bénéficié de formation relative au tutorat d'enseignant ; Paul, tuteur ESPE, âgé de 52 ans, exerçait depuis vingt ans le métier de formateur universitaire (actuellement formateur dans le Master Métiers de l'éducation, de l'enseignement et de la formation, parcours EPS) ; il effectuait pour la deuxième année consécutive des visites formatives au sein du dispositif du tutorat mixte. La tutrice et le tuteur n'avaient jamais travaillé ensemble. Le professeur stagiaire, lauréat du concours, était âgé de 24 ans.

Binôme 2: Delphine, enseignante en éducation physique et sportive, âgée de 48 ans, accueillait depuis trois ans des stagiaires de différents niveaux (Licence et Master) sans avoir bénéficié de formation relative au tutorat d'enseignant ; Valérie, tutrice ESPE, âgée de 57 ans, exerçait depuis 25 ans le métier de formatrice universitaire (actuellement formatrice dans le Master Métiers de l'éducation, de l'enseignement et de la formation, parcours EPS). Depuis deux ans, elle assurait les visites formatives dans le cadre du tutorat mixte. Les deux tutrices avaient déjà eu l'occasion de travailler ensemble dans le cadre d'une commande institutionnelle sur la rédaction des fiches ressources académiques en éducation physique. Le professeur stagiaire était âgé de 25 ans.

3. 3. Recueil des données d'étude

Dans cette étude, trois types de données ont été recueillis au cours de l'année 20152016 : (a) des données d'enregistrement audiovisuel de l'activité des tuteurs lors d'une visite formative ; (b) des données d'entretien d'autoconfrontation simple (ACS) avec chaque tuteur ; (c) enfin, des données de confrontation collective (CC) rassemblant les deux binômes autour des matériaux issus des analyses du chercheur sur les données d'ACS. En s'écartant de la démarche « canonique » mise en œuvre en Clinique de l'activité (Clot, 2008 ; Clot, Faïta, Fernandez \& Scheller, 2000; Yvon \& Clot, 2003), le dispositif a du répondre d'une part aux contraintes de disponibilités des tuteurs tout en conciliant les exigences d'une méthode en deux temps avec une continuité des échanges

\footnotetext{
${ }^{2}$ Professeur stagiaire : étudiant lauréat du concours CAPEPS, inscrit en deuxième année du Master MEEF
} 


\section{eJRIEPS 41 avril 2017}

(Faïta \& Vieira, 2003) et d'autre part aux caractéristiques de l'activité collective du tutorat mixte, un binôme «Tuteur ESPE- Tuteur EPLE » confronté à un autre binôme, en présence du chercheur. Un tel aménagement a pu respecter le cadre général de la coanalyse mis en place dans une autoconfrontation croisée entre un professionnel et un pair.

(a) L'enregistrement audiovisuel de la visite formative réalisée par chaque binôme a été effectué à l'aide d'une caméra numérique mobile et d'un micro HF porté par chaque tuteur. La durée d'enregistrement de deux heures trente comprenait le travail d'observation des deux tuteurs lors de la leçon menée par le stagiaire et l'activité d'entretien des deux tuteurs avec le stagiaire. Pour le binôme 1, l'observation du 6 novembre a porté sur la troisième leçon de relais-vitesse sur un cycle de 9 leçons, avec 28 élèves d'une classe de première Economique et Sociale. La pratique a eu lieu sur un espace relativement étendu, constitué de 6 couloirs et délimité par la ligne de départ et d'arrivée du 100 mètres sur le stade d'athlétisme. Pour le binôme 2, les deux tuteurs ont observé, le 21 novembre, la $5^{\text {ème }}$ leçon de natation sur un cycle de 8 séances, avec une classe de 24 élèves de $6^{\text {ème }}$. L'espace de pratique était délimité par trois lignes d'eau d'un bassin de 25 mètres.

Quant à l'entretien mené par chaque binôme avec le stagiaire, il a eu lieu dans une salle de l'établissement scolaire, une heure après le travail d'observation. Ce délai correspondait à la durée du trajet retour (de l'installation sportive à l'établissement). Au cours de ce trajet, les deux binômes ont un peu échangé sur leurs observations et l'organisation de l'entretien.

(b) Les entretiens d'autoconfrontation simple ont été réalisés en décembre, un mois après les enregistrements. Chaque tuteur a été confronté au moins à trois extraits vidéo sur le travail d'observation et trois extraits vidéo sur l'entretien avec le stagiaire. Les extraits vidéo, d'une durée de quatre à sept minutes, ont été sélectionnés par le chercheur à partir des réactions spontanées des tuteurs recueillies à l'issue de la visite formative; par exemple, «l'observation de tout à l'heure en fait je savais pas trop quoi faire par rapport au tuteur », " lors de l'observation, j'ai complétement fait abstraction de ma collègue tutrice », «c'était dur de savoir à quel moment il fallait que je prenne la parole j'ai hésité longtemps à la prendre », « lors de l'entretien, je n'ai pas compris pourquoi il lui a dit ça ». À chaque entretien, un accord a été obtenu auprès des tuteurs quant aux choix des extraits vidéo et aux « réactions » respectives que ces extraits suscitaient chez eux. Le chercheur a accompagné chaque tuteur (confronté pour la première fois à la méthode des autoconfrontations) à exprimer précisément les difficultés du travail collectif vécue lors de 


\section{eJRIEPS 41 avril 2017}

la visite formative. Les questions posées lors de ces entretiens d'autoconfrontation simple pouvaient s'énoncer ainsi : “Avant d'observer, tu t'étais mis d'accord avec le tuteur sur une procédure ? Tu peux préciser pourquoi tu as hésité à intervenir en présence de l'autre tuteur? En quoi ces actions te paraissaient importantes pour la collaboration avec la collègue tutrice ? Te semblaient-elles contradictoires ? C'est toi qui as décidé de te mettre en retrait lors de l'entretien post-leçon? ».

(c) L'objectif des deux confrontations collectives était de proposer aux tuteurs/trices ESPE et EPLE, deux mois après les entretiens d'autoconfrontation simple, un nouveau contexte d'échange et d'analyse des conflits inhérents à l'activité collective. D'une durée d'une heure trente à deux heures, ces confrontations ont permis dans un premier temps de restituer aux tuteurs les conflits identifiés à travers la dimension contrariée de l'activité collective et dans un deuxième temps d'inviter les tuteurs à échanger sur ces conflits pour construire ensemble une nouvelle signification. Ces confrontations collectives ont été enregistrées puis retranscrits intégralement.

\section{3. Traitement des données}

Dans un premier temps, le corpus constitué des quatre entretiens d'autoconfrontation simple a été découpé en unités d'analyse délimitées à partir de l'énonciation, par l'acteur, d'une activité contrariée. Dans les verbatim correspondant à l'unité, en référence à la macrostructure de l'activité humaine (Leontiev, 1984), ont été identifiés les énoncés en lien avec le but (action), les motifs (ce qui oriente l'activité) et les opérations (moyens permettant d'atteindre le but).

Dans un second temps, un protocole à quatre volets a été construit : le premier volet présentait la transcription verbatim des entretiens d'autoconfrontation simple et l'identification des composantes de l'activité (but d'action, motif et opération) ; le second volet rassemblait les énoncés permettant d'identifier un premier niveau de formalisation du conflit de l'activité collective ; le troisième volet comprenait la transcription verbatim des confrontations collectives en correspondance avec le conflit identifié ; le quatrième volet présentait les énoncés permettant d'identifier un second niveau de formalisation plus générique du conflit. Le tableau 1 présente la procédure d'analyse des données. 


\section{eJRIEPS 41 avril 2017}

Tableau 1. Illustration du traitement des données d'entretien.

\section{Volet 1}

Transcription Autoconfrontation simple avec Anna (tutrice EPLE)

Anna : en fait on est arrivés ensemble, on a du se dire deux mots et après P. (tuteur ESPE) s'est écarté et effectivement dès le départ, je ne savais pas quelle posture adoptée, je ne savais pas si on devait être côte à côte pour échanger régulièrement ou si on devait garder une certaine distance, mais dès le départ, $P$. (tuteur ESPE) a adopté une posture plus éloignée de moi dès le début de la séance, on le voit ici il est assez loin donc je me suis dit «tiens, comme je suis nouvelle tutrice c'est la posture à adopter " c'est à dire neutralité, chacun de son côté, on ne s'influence pas, on ne dialogue pas

Identification des composantes de l'activité

But d'action

Observer

\section{Motif}

échanger régulièrement

ne pas dialoguer

\section{Opération}

en étant côte à côte

en gardant une certaine distance

Transcription Autoconfrontation simple avec Paul (tuteur ESPE)

Chercheur : alors c'est le début de l'observation et tu préfères te placer ici assez loin en fait de ta collègue

Paul (Tuteur ESPE): oui alors je me suis placé de telle sorte à filmer $C$ (stagiaire) pas trop près pour ne pas gêner les élèves et le stagiaire et ces prises vidéos je les ai utilisées ensuite dans l'entretien (...)mais c'est vrai maintenant qu'on voit ça je me dis que ma façon de faire a peut-être intrigué la tutrice en fait pour moi comme je venais pour le stagiaire j'ai même pas pensé à échanger avec la tutrice

Identification des composantes de l'activité

But d'action

Observer

\section{Motif}

filmer le stagiaire échanger
Opération

se placer...pas trop près

\section{Volet 2}

Premier niveau de formalisation du conflit de l'activité collective:

Observer en étant côte à côte pour échanger / en gardant une certaine distance de telle sorte à ne pas dialoguer

Echanger / filmer

\section{Volet 3}

Transcription Confrontation collective avec les quatre tuteurs

Valérie (Tutrice ESPE) : nous pendant l'observation on était assez proches pour échanger mais avec Delphine (Tutrice EPLE) on n'a pas échangé sur ce qu'on voyait, on a parlé du métier (...) alors de mon point de vue notre travail d'observation à toutes les deux c'est ni la distance, une posture qui pourrait être neutre, ni le côte à côte pour échanger sur l'activité du stagiaire, la question est plutôt de savoir ce qu'on y fait dans cette observation et dans ces échanges et pour nous c'était un espace de partage des éléments contextuels

Delphine (Tutrice EPLE) : on a échangé sur le contexte d'enseignement général

Paul (Tuteur ESPE) j'avais besoin d'échanger par rapport à ce que je voyais et d'autres moments de la séance j'ai eu besoin de prendre de la distance (...) le véritable dilemme c'est peut-être pas celuilà mais plutôt le dilemme qui me pousse à choisir entre obtenir un consensus sur les observables sur lesquels je vais mettre la focale qui sont les plus fondamentaux à ce moment là pour le stagiaire et retenir tous les éléments qui sont pour moi important à ce moment là

\section{Volet 4}

Deuxième niveau de formalisation du conflit

Pendant l'observation du stagiaire,

Échanger avec son collègue tuteur : Echanger sur l'activité du stagiaire / Échanger sur le métier

Recueillir des traces sur l'activité du stagiaire : Choisir les observables fondamentaux pour le stagiaire / retenir tous les éléments de l'activité important pour le tuteur 


\section{eJRIEPS 41 avril 2017}

\section{Résultats}

Les résultats présentent deux conflits portant sur l'activité collective du tuteur ESPE et du tuteur EPLE, pendant l'observation et l'entretien post-leçon. Le choix de ces deux conflits se justifie par la richesse des controverses favorisant le développement par le collectif des tuteurs d'une formalisation des conflits. Les différents extraits d'entretien d'autoconfrontation simple et de confrontation collective illustrent l'intérêt des échanges inter-psychiques comme espace d'émergence et d'élaboration de nouvelles significations à propos des conflits sur l'activité collective des tuteurs ESPE et EPLE.

4. 1. Pendant l'observation, échanger avec le tuteur / recueillir les traces de l'activité du stagiaire

$\mathrm{Au}$ cours de l'entretien d'autoconfrontation, Anna, nouvelle tutrice en établissement, a décrit les différentes actions réalisées lors de la séquence d'observation du stagiaire: « noter successivement sur une feuille les interventions du stagiaire et les comportements des élèves (actions) pour en reparler plus tard dans l'entretien et avoir des exemples concrets à dire au stagiaire (motifs) ». Elle a expliqué qu'elle s'était référée à une expérience vécue lors de la visite de l'inspecteur (je pense que j'ai un peu reproduit ce que j'avais vécu y'a pas si longtemps d'ailleurs cette fameuse inspection où je vois encore l'inspecteur se mettre sur le côté et prendre des notes). Anna a ensuite précisé à quel point la posture d'observateur de son collègue tuteur l'avait intriguée (Paul s'est écarté et effectivement dès le départ je ne savais pas si on devait être côte à côte pour échanger régulièrement ou si on devait garder une certaine distance).

\section{Extrait 1 (Autoconfrontation simple avec Anna)}

Chercheur : Là tu es seule en train de regarder le stagiaire, tu es placée assez loin de Paul (tuteur), tu prends des notes, tu te déplaces cette façon d'observer l'intervention du stagiaire c'est toi qui avais décidé de travailler comme ça

Anna (Tutrice EPLE) : en fait on est arrivé ensemble, on a du se dire deux mots et après $P$. s'est écarté et effectivement dès le départ, je ne savais pas quelle posture adoptée, je ne savais pas si on devait être côte à côte pour échanger régulièrement ou si on devait garder une certaine distance, mais dès le départ, $P$. (tuteur) a adopté une posture plus éloignée de moi dès le début de la séance, on le voit ici il est assez loin donc je me suis dit «tiens, comme je suis nouvelle tutrice c'est la posture à adopter " c'est à dire neutralité, chacun de son côté, on ne s'influence pas, on ne dialogue pas et justement lors de l'entretien on verra les choses qu'on a analysées l'un et l'autre mais en fait entre observer ensemble, se créer comme un espace de co-observation et adopter une posture d'observateur isolé en restant éloignés je ne savais pas ce qu'il fallait faire

Chercheur: tu veux dire que tu n'en as pas parlé avec le tuteur et arrivée sur le stade tu t'es posée cette question de la posture à adopter lors de l'observation

Anna : oui je me suis dit « on est là ensemble mais on ne va peut-être pas se rejoindre durant l'observation » 


\section{eJRIEPS 41 avril 2017}

Dès le début du travail avec son collègue tuteur, la tutrice a questionné la posture d'observateur pour laquelle elle a énoncé deux motifs et deux opérations concurrentes (observer en étant proche de telle sorte à échanger régulièrement / observer chacun de son côté pour ne pas s'influencer). Etant nouvelle tutrice, elle a pensé que son tuteur adoptait «la bonne posture», une posture éloignée pour ne pas dialoguer (motif) et qu'elle n'avait pas d'autres choix que de s'y tenir. De son côté, confronté au même extrait vidéo, Paul a expliqué qu'il était « venu voir le stagiaire et uniquement le stagiaire (action) pour prendre un maximum d'informations sur son intervention » (motif). II a ensuite convoqué l'expérience des autres visites formatives pour justifier l'usage d'un appareil enregistreur des traces de l'activité du stagiaire. Sur ce point, il a évoqué une posture ajustée pour ne pas gêner les élèves et le stagiaire (motif). Mais au cours de l'entretien, Paul a manifesté progressivement une certaine réserve vis à vis des actions réalisées lors de la phase d'observation.

\section{Extrait 2 (Autoconfrontation simple avec Paul)}

Chercheur: alors c'est le début de l'observation et tu préfères te placer ici assez loin en fait de ta collègue

Paul (Tuteur ESPE): oui alors je me suis placé de telle sorte à filmer $C$ (stagiaire) pas trop près pour ne pas gêner les élèves et le stagiaire et ces prises vidéos je les ai utilisées ensuite dans l'entretien

Chercheur: vous aviez échangé tous les deux sur cette manière de recueillir des traces vidéo de l'activité du stagiaire

Paul : non non mais pour moi c'était important d'avoir ces traces pour soulever des points problématiques, des situations critiques en fait ce sont les ingrédients de l'entretien que je construis moi-même je le fais quasiment dans toutes les visites mais c'est vrai maintenant qu'on voit ça je me dis que ma façon de faire a peut-être intrigué la tutrice en fait pour moi comme je venais pour le stagiaire j'ai même pas pensé à échanger avec la tutrice sur ce qu'on allait faire pendant la séance de $\mathrm{C}$.

Le tuteur ESPE a expliqué qu'il avait organisé son activité selon le protocole habituel consistant à « filmer (action) pour utiliser les traces vidéos dans l'entretien (motif) 》. II a clairement souligné l'intérêt de construire ces traces envisagées comme les ingrédients de l'entretien. Plus loin, on comprend qu'il prend conscience au moment où il en parle des limites de ses actions (c'est vrai maintenant qu'on voit ça je me dis que ma façon de faire a peut-être intrigué la tutrice). Ses commentaires ont notamment évoqué le fait de ne pas avoir discuté avec la tutrice du travail d'observation.

Plus tard, la confrontation collective va permettre aux quatre tuteurs de discuter de leur posture d'observateur à travers le conflit identifié par le chercheur. 
Extrait 3 (Confrontation collective avec les quatre tuteurs)

Chercheur: pour votre travail d'observation, j'ai relevé ce conflit entre " échanger avec son collègue » et "recueillir des traces de l'activité du stagiaire » alors je voudrais que vous me précisiez ce conflit, Anna m'avait dit que pour elle c'était un dilemme alors qu'est-ce que vous en pensez est-ce que vous avez été confrontés au même conflit ou dilemme pendant l'observation

Valérie (Tutrice ESPE) : nous pendant l'observation on était assez proches pour échanger mais avec Delphine (Tutrice EPLE) on n'a pas échangé sur ce qu'on voyait, on a parlé du métier, de l'enseignement de la natation, du choix de l'équipe pédagogique et pas de l'activité du stagiaire mais ces échanges étaient pour moi aussi importants parce qu'ils me permettaient de comprendre ce jour là pourquoi le stagiaire proposait tel contenu (...) alors de mon point de vue notre travail d'observation à toutes les deux c'est ni la distance, une posture qui pourrait être neutre, ni le côte à côte pour échanger sur l'activité du stagiaire, la question est plutôt de savoir ce qu'on y fait dans cette observation et dans ces échanges et pour nous c'était un espace de partage des éléments contextuels

Delphine (Tutrice EPLE) : oui oui, toutes les deux, on a échangé sur le contexte d'enseignement général

Paul (Tuteur ESPE): alors moi par rapport à ce dilemme c'est vrai qu'il y a eu des moments où j'avais besoin d'échanger par rapport à ce que je voyais et d'autres moments de la séance j'ai eu besoin de prendre de la distance, de me reculer, d'être seul parce que j'avais besoin de faire le point mais pour moi c'est pas un dilemme car à aucun moment j'ai eu l'impression d'hésiter entre les deux je comprends plutôt que j'ai alterné les deux actions sans que cela me pose de problème

Chercheur : Et toi Anna tu veux réagir?

Anna (Tutrice EPLE): alors ce dilemme, oui je l'ai vécu, et en même temps je ne l'ai pas mal vécu car je comprenais pourquoi tu étais éloigné et ton outil effectivement nous éloignait aussi j'allais pas me rapprocher et échanger avec toi alors que tu filmais ; mais moi je me dis après coup que dialoguer avant, ça m'aurait permis de mieux comprendre certaines choses étant nouvelle tutrice

Cet extrait permet d'apprécier l'intérêt du dialogue collectif comme moyen de relancer la discussion autour des actions à l'origine d'un conflit. Valérie n'a pas hésité à préciser le contenu des échanges avec sa collègue tutrice et le motif d'action partagé (on n'a pas échangé sur ce qu'on voyait, on a parlé du métier (...) pour comprendre ce jour là pourquoi le stagiaire proposait tel contenu - motif). Elle a également reconsidéré le travail d'observation en le définissant comme un espace de partage des éléments contextuels qu'elle a pu mettre en place avec sa collègue tutrice. Elle a ensuite inscrit l'activité d'observation dans un questionnement plus général (c'est ni la distance, une posture qui pourrait être neutre, ni le côte à côte pour échanger sur l'activité du stagiaire, la question est plutôt de savoir ce qu'on y fait dans cette observation et dans ces échanges). Paul a poursuivi le dialogue en parlant d'alternance entre une posture de proximité avec sa collègue et une mise en retrait. Le souvenir de son expérience vécue lors du travail 


\section{eJRIEPS 41 avril 2017}

d'observation lui a permis de ne pas considérer cette alternance à l'origine d'un dilemme. Le dialogue a ensuite conduit Anna à reprendre l'énoncé du dilemme en précisant que l'éloignement de Paul n'avait pas engendré d'inconfort. Elle a ensuite affirmé l'utilité pour une tutrice novice d'échanger avant la visite formative afin de mieux comprendre la situation de travail.

Plus loin dans l'échange, Paul est revenu sur l'importance de recueillir des traces sur l'activité du stagiaire.

Extrait 4 (Confrontation collective avec les quatre tuteurs)

Paul (Tuteur ESPE) : le véritable dilemme c'est peut-être pas celui-là mais plutôt le dilemme qui me pousse à choisir entre obtenir un consensus sur les observables sur lesquels je vais mettre la focale qui sont les plus fondamentaux à ce moment là pour le stagiaire et retenir tous les éléments qui sont pour moi important à ce moment là et à ce dilemme j'en vois un autre plus général c'est cibler sur ce qui est essentiel pour lui à ce moment là de sa carrière parce que c'est ça dont il a besoin et pas autre chose ou balayer l'activité du stagiaire le plus largement possible ce n'est pas du tout la même chose mais en fait une des solutions serait de se dire qu'il faut réussir au moins à se mettre d'accord à trois avec le stagiaire sur un projet de formation partagé

La confrontation collective a été l'occasion pour Paul de poursuivre la discussion autour du conflit identifié par le chercheur en exposant un point de vue différent de celui énoncé par Anna (le véritable dilemme c'est peut-être pas celui-là). II a ainsi formalisé un autre dilemme propre à son activité en évoquant deux actions contradictoires, l'action de « choisir les observables fondamentaux pour le stagiaire » et celle de « retenir tous les éléments importants (pour moi)». II a proposé une formalisation plus générale (à ce dilemme j'en vois un autre plus général) ouverte sur une préoccupation constante entre l'action de « cibler les observations essentielles pour le stagiaire et celle de balayer l'activité du stagiaire ». Paul a ensuite énoncé un motif susceptible d'être partagé par ses collègues pour résoudre la contradiction des actions (se mettre d'accord à trois avec le stagiaire sur un projet de formation partagé).

A travers l'extrait, on comprend que le dialogue entre les tuteurs a favorisé l'énonciation de certains empêchements ou difficultés professionnelles vécues lors de l'observation. L'expression de ces difficultés a parfois conduit les tuteurs à juger de la pertinence du conflit tout en faisant évoluer le sens de l'activité d'observation ; au gré des arguments, le conflit est devenu un objet de discussion ouvert sur une formalisation plus générique. 


\section{eJRIEPS 41 avril 2017}

4. 2. Au cours de l'entretien, faire un débriefing avec le stagiaire / construire une relation complémentaire

Confrontée aux images de l'entretien post-leçon, Delphine a expliqué qu'elle s'était rendue compte de la difficulté à se positionner vis à vis du stagiaire et de sa collègue. Elle a précisé avoir commencé l'entretien en s'adressant au stagiaire «comme dans un débriefing ». A travers l'extrait 5, on comprend que n'ayant pas eu de concertation préalable avec sa collègue, Delphine s'est engagée dans l'entretien selon des modalités d'échange construites avec le stagiaire depuis la rentrée scolaire.

Extrait 5 (Autoconfrontation simple avec Delphine)

Delphine (Tutrice EPLE) : je me suis demandé comment on l'abordait cet entretien je me suis dit on s'est pas concertées pour voir comment on allait s'y prendre comment on allait l'organiser et comment on allait le mener etc et Valérie nous a demandé comment on faisait d'habitude et du coup j'ai commencé en lui posant une question comme dans un débriefing «qu'est-ce que t'as pensé de ta séance ?» Chercheur : tu veux dire que vous avez organisé l'entretien comme tu as l'habitude de le faire toute seule avec le stagiaire

Delphine : oui oui et après on a embrayé et là comme c'est un garçon (stagiaire) qui doute beaucoup quand Valérie prend la parole en fait je me dis qu'elle ne lui dit pas ce que moi je lui dis toujours dans les débriefing pour le rassurer ; on peut dire qu'à ce moment là j'ai un peu mal pour lui ; (...) en fait pendant l'entretien je réalise que d'un côté j'ai le stagiaire avec qui j'ai construit des choses et de l'autre côté la tutrice avec qui je dois construire un autre dialogue

Delphine a ainsi expliqué qu'elle aurait souhaité voir la tutrice ESPE s'entretenir différemment auprès du stagiaire. Elle a précisé qu'elle avait pris conscience durant l'entretien que l'action réalisée par la tutrice ne pouvait pas garantir du moins favoriser ce qu'elle tentait de faire depuis la rentrée scolaire pour rassurer le stagiaire (motif). La manière dont elle a décrit la situation laisse penser que Delphine a été confrontée à un conflit entre deux actions concurrentes, celle de poursuivre le travail de débriefing avec le stagiaire et celle de construire une relation avec son collègue tuteur.

La tutrice ESPE a confirmé cette situation problématique lorsqu'elle a décrit l'entretien réalisé avec la tutrice EPLE et le stagiaire.

Extrait 6 (Autoconfrontation simple avec Valérie)

Chercheur: avant de rentrer dans l'entretien, vous vous étiez mis d'accord sur certains principes ?

Valérie (Tutrice ESPE) : on s'est mis d'accord seulement sur la durée de l'entretien mais on s'est pas mis d'accord sur le cadre et je dois dire que ça me pose problème

Chercheur : ça te pose problème de ne pas en avoir parlé avec ta collègue tutrice Valérie : pas plus que ça mais y'a autre chose parce que l'entretien il a démarré au bord du bassin pour moi et j'aime bien que l'entretien parte du stagiaire je trouve 


\section{eJRIEPS 41 avril 2017}

que c'est bien (...) ensuite quand je parle à B. (stagiaire) je vois bien que Delphine se retourne vers moi pour me signifier quelque chose

Chercheur: son regard te gêne?

Valérie : oui en gros, son regard me dit tu ne vas pas lui reprocher et je vois bien que là dans l'entretien j'ai en face de moi un duo Delphine et son stagiaire et j'ai l'impression que je suis perçue extérieure à ce duo alors qu'on est co- tutrices ; en fait elle fait un peu comme les autres tuteurs en établissement qui se justifient pour un peu défendre le stagiaire et dans ce cas je suis un peu le tiers exclu (...)

Valérie a tout d'abord souligné dans l'autoconfrontation l'absence d'un accord à propos du cadre de l'entretien post-leçon. Puis elle a confirmé le malaise occasionné par la situation d'échange, en évoquant particulièrement le regard de Delphine, l'impression de face à face avec un duo «Tutrice-Stagiaire » et ce sentiment d'être « la tierce personne exclue ». Elle en a rappelé la permanence dans les visites formatives « elle fait un peu comme les autres tuteurs en établissement qui se justifient pour un peu défendre le stagiaire ».

Lors de la confrontation collective, la relation «tutrice-stagiaire » et le positionnement de chacun dans l'entretien post-leçon font l'objet d'un nouvel échange.

\section{Extrait 7 (Confrontation collective avec les quatre tuteurs)}

Chercheur: vous avez rencontré des difficultés à vous situer l'un par rapport à l'autre pendant l'entretien post-leçon; pour Delphine et Anna la difficulté a surtout été de passer du face à face avec le stagiaire à un échange à trois, Delphine toi tu avais même parlé d'un dilemme entre l'action de rester marraine du stagiaire et celle de devenir partenaire du formateur ESPE, j'aimerais que vous en reparliez

Paul (Tuteur ESPE): en fait le dilemme il nous dit aussi que moi tuteur quand je vais rendre visite il faut que je me fonde dans une relation, la relation de la tutrice et le stagiaire ; et en entretien c'est pareil et c'est pas forcément facile ça suppose qu'on la sente cette relation

Chercheur: et une fois que tu es rentré dans cette relation tutrice-stagiaire tu penses avoir construit une autre relation (-)

Valérie : il faudrait que ce soit un entretien circulaire pour éviter le « deux contre un »

Delphine (Tutrice EPLE): moi par rapport au dilemme alors je reviens un peu sur ce que j'avais dit l'autre jour je crois que ce rôle que j'ai dans l'entretien être marraine du stagiaire je pense maintenant qu'on peut à la fois être marraine du stagiaire tout en étant partenaire de la collègue tutrice parce qu'on est complémentaire (-)

Valérie : j'allais le dire

Anna (Tutrice EPLE): alors complémentaire oui et je me souviens pour nous deux que lorsque tu rebondissais sur ma question je me disais que j'étais dans le juste questionnement

Valérie : en fait dans l'entretien on a tous les deux cette posture qui se veut à la fois étayée entre nous et étayant vis à vis du stagiaire

Au cours de la confrontation collective, Paul a parlé du dilemme en évoquant notamment la difficulté de s'immerger dans une organisation et un partenariat déjà établi entre le 


\section{eJRIEPS 41 avril 2017}

tuteur EPLE et le stagiaire. II a confirmé le fait que cette relation dyadique « tuteur EPLE et stagiaire » était un élément à prendre en considération lors des visites formatives, en précisant une manière (opération) d'optimiser l'immersion (il faut que je me fonde dans une relation). Pour Delphine, le fait de reparler de cette relation lui a permis d'évoquer un nouveau motif, celui de concilier (et non plus d'opposer) le partenariat avec le stagiaire et celui avec le tuteur pour créer une complémentarité. D’ailleurs, chacune leur tour, Anna puis Valérie ont évoqué les opérations favorables au développement d'une complémentarité des rôles entre les tuteurs et avec le stagiaire (« rebondir sur les questions du collègue », « avoir une posture étayante et étayée »). Au final, les tuteurs ont tous exprimé, de façon plus ou mois explicite, le motif de ne pas faire reposer l'entretien sur une relation de « deux contre un ».

A travers cet extrait, on constate que le conflit identifié par le chercheur fait écho aux difficultés que les tuteurs ESPE et les tuteurs EPLE ont rencontrées lors de l'entretien post-leçon. La signification du conflit trouve ainsi dans le dialogue l'occasion d'être développée à travers l'expression d'affects (sentir la relation tutrice-stagiaire pour s'y fondre), l'évocation de l'expérience passée (je me souviens pour nous deux que lorsque tu rebondissais sur ma question...) et l'élaboration d'un nouveau sens quant au positionnement de chacun lors de l'entretien post-leçon (être complémentaire).

\section{Discussion des résultats et perspectives de formation des tuteurs ESPE et EPLE}

Si les résultats mériteraient d'être complétés par l'analyse des autres conflits et par d'autres données d'étude, ils ont toutefois permis de documenter l'activité contrariée des tuteurs ESPE et EPLE impliqués dans le travail du tutorat mixte. A l'appui de ces résultats, les contraintes des mises en œuvre de ce nouveau dispositif d'accompagnement (Rapport IGAENR, 2015) trouvent ici des explications possibles. Les résultats permettent d'aborder deux points de discussion.

Le premier point de discussion concerne le développement de la signification du conflit au cours de la confrontation collective. L'analyse des données d'étude montre la capacité des tuteurs à s'approprier individuellement et collectivement les dimensions contrariées de l'activité pour élaborer une nouvelle signification de leur expérience. En introduisant la controverse à partir de l'énoncé d'un conflit et/ou d'un dilemme, le chercheur a ainsi incité les binômes «Tuteur ESPE-Tuteur EPLE » à préciser ce qui n'avait pas été encore dit sur le conflit et à analyser autrement et ensemble l'activité. Lors des confrontations collectives, le passage d'un premier niveau de formalisation à un deuxième niveau de formalisation du 


\section{eJRIEPS 41 avril 2017}

conflit s'est réalisé au travers de liaisons avec les circonstances de l'expérience passée mais aussi au contact des formes d'interprétation dissonantes sur le conflit. Ce résultat confirme l'intérêt de relancer le processus de formalisation des conflits en déplaçant un conflit intrapsychique dans l'inter-psychique (Bertone \& al, 2003 ; Moussay, 2012). Dans le cas de l'activité d'entretien réalisée par les deux tuteurs, la formalisation du conflit « faire un débriefing avec le stagiaire / construire un dialogue avec le tuteur " s'est développée à travers de nouveaux énoncés de motifs : "se fondre dans la relation dyadique ", "éviter le deux contre un" "construire une complémentarité de regards". Par ailleurs, le dialogue entre les tuteurs a favorisé l'expression de différents points de vue faisant émerger des formes d'énonciation et de problématisation du conflit tantôt singulières en lien avec l'expérience individuelle tantôt génériques à l'image de ce que Delphine a évoqué sur le positionnement de chacun au sein de la triade («on peut à la fois être marraine du stagiaire tout en étant partenaire de la collègue tutrice parce qu'on est complémentaire »). Pour le dire autrement, les conflits dont la signification s'était figée en une version univoque et souvent trop personnalisée (Scheller, 2013) ont pu par le dialogue s'ouvrir vers une nouvelle signification partagée et collective et traduire un problème de métier.

A travers ce premier point de discussion, on comprend l'utilité de mettre en place des confrontations plus systématiques lors desquelles les tuteurs ESPE et EPLE pourraient se mettre d'accord sur les significations attribuées au travail de l'enseignant novice (Bertone \& Chaliès, 2015) mais également sur les significations à propos des empêchements, des difficultés, des actions concurrentes et/ou contradictoires du travail. A cet égard, penser la formation des tuteurs autour des outils de confrontation collective et de co-analyse du travail apparaît aujourd'hui comme une piste pour répondre aux enjeux du travail collectif du tutorat mixte (Moussay, 2016) et aboutir à la constitution de collectifs de formateurs (Chaliès, 2015).

Le deuxième point de discussion porte sur la tension entre les significations que les tuteurs ont exprimées à propos des énoncés de conflits. Comme on a pu le constater avec Paul, celui-ci a interrogé les actions et les motifs vécus comme contradictoires par sa collègue tutrice, tout en affirmant qu'ils ne trouvaient pas d'écho dans sa pratique. En d'autres termes, sa perception différente de la situation d'observation a relancé la controverse sur la dimension contrariée de l'activité, favorisant ainsi l'émergence d'un nouveau dilemme (cibler les observations qui sont essentielles pour le stagiaire / balayer l'activité du stagiaire). Ce constat permet de mettre en avant l'apport du dialogue collectif 


\section{eJRIEPS 41 avril 2017}

comme moyens d'adresser aux pairs, mais aussi au chercheur, de nouveaux énoncés de conflit. Enfin, les résultats ont montré que la mise en tension des significations sur la dimension contrariée de l'activité avait permis aux tuteurs d'exprimer à l'égard du conflit des sentiments de malaise, d'inconfort, de mise à l'épreuve, de regrets, d'impuissance. Dans ce cas, la confrontation collective a créé un autre espace de développement conceptuel sur l'activité contrariée au delà du concept de conflit et des analyses proposées par le chercheur. En ce sens, le dialogue n'a pas seulement favorisé le développement de la signification des conflits mais il a également permis une évolution de la signification des affects. Par exemple, le sentiment d'inconfort exprimé par Anna lors de l'entretien d'autoconfrontation a évolué selon ses propos "le dilemme oui je l'ai vécu, et en même temps je ne l'ai pas mal vécu car je comprenais pourquoi tu étais éloigné ".

L'essentiel de ce deuxième point de discussion concerne la mise en place de situations de formation ouverte sur le dialogue entre le tuteur ESPE et le tuteur EPLE afin de favoriser le développement des conceptualisations. A travers ces situations, l'objectif serait de provoquer des mises en tension entre les propres analyses des tuteurs sur leur expérience du travail et les outils conceptuels (tels les conflits) pour les aider à élaborer un nouveau sens de leur activité collective. Ce processus d'élaboration pourrait également favoriser la migration des affects de la sphère personnelle à la sphère collective du tutorat mixte.

En conclusion, cette étude permet d'entrevoir de nouvelles priorités pour la formation de la dyade “Tuteur ESPE et Tuteur EPLE » en éducation physique et sportive mais aussi dans les autres disciplines. Ces priorités accordées au collectif de formateurs et aux échanges intersubjectifs entre les deux tuteurs s'inscrivent dans la perspective d'une autre priorité, celle de l'accompagnement des fonctionnaires stagiaires dans l'analyse réflexive de leur activité (Bertone, Chaliès, \& Clot, 2009). En ce sens, le développement du collectif «Tuteur ESPE - Tuteur EPLE » peut contribuer à deux apports majeurs dans la formation professionnelle des enseignants : d'une part, un apport concernant la prise en charge collective des préoccupations, voire des conflits vécus par les stagiaires, pour construire un projet de formation partagé (Gaudin, Perrot, Chaliès, Escalié, \& Raymond, 2015) ; d'autre part, un apport concernant la mise en place d'un espace d'analyse croisée du travail (confrontation entre les tuteurs) et de discussion professionnelle pour enrichir le point de vue du stagiaire et l'aider à prendre des décisions face aux différentes manières de faire le métier. 


\section{eJRIEPS 41 avril 2017}

\section{Bibliographie}

Balas, S. (2011). Le référentiel, un outil de formation, un instrument de développement du métier. Le métier de masseur-kinésithérapeute en référence. Thèse de doctorat de sciences de l'éducation. Paris: CNAM, http://tel.archives-ouvertes.fr/tel-00669690

Bertone, S., Méard, J.A., Ria, L., Euzet, J.P., Durand, M. (2003). Intrapsychic conflict experienced by a preservice teacher during classroom interactions : A case study in physical education. Teaching and Teacher Education, 19, 1, 113-125.

Bertone, S., Chaliès, S., \& Clot, Y. (2009). Contribution d'une théorie de l'action à la conceptualisation et à l'évaluation des pratiques réflexives dans les dispositifs de formation intiale des enseignants. Le travail Humain, 72, 2, 104-125.

Bertone, S., \& Saujat, F. (2013). La réflexivité comme instrument de formation par l'alternance et de développement de l'activité professionnelle des enseignants. @ctivités, 10, 2, 177-192.

Bertone, S., \& Chaliès, S. (2015). Construire un programme de recherche technologique sur la formation des enseignants: choix épistémologiques et théoriques», @ctivités, 12, 2.

Boutet, M., \& Pharand, J. (Dir.) (2008). L'accompagnement concerté des stagiaires en enseignement. Québec : Presses Universitaires du Québec.

Chaliès, S. (2015). Activités typiques et dilemmes des formateurs d'enseignants. In V. Lussi-Borer \& L. Ria (Eds.), Apprendre à enseigner, (pp. 221-233). Paris : Presses Universitaires de France.

Chaliès, S., Bruno, F., Méard, J., \& Bertone, S. (2010). Training preservice teachers rapidly: the need to articulate the training given by university supervisors and cooperating teachers. Teaching and Teacher Education, 26, 764-774.

Chaliès, S., Escalié, G., Bertone, S. (2012). Etude d'un travail collaboratif de formation professionnelle initiale des enseignants : résultats et propositions. Savoirs, 29, 5978.

Chambers, F. C., \& Armour, K. (2012). School-university part- nerships and physical education teacher education student learning: A fruitful division of labour? European Physical Education Review, 18, 159-181.

Ciavaldini-Cartaut, S. (2016). Multimodalité numérique en supervision de stage. Education permanente, 206, 61-74.

Ciavaldini-Cartaut, S. (2012). Innover en formation. Accompagner autrement les enseignants entrant dans le métier. Paris : L'Harmattan. 


\section{eJRIEPS 41 avril 2017}

Clot, Y. (1999). La fonction psychologique du travail. Paris: Presses Universitaires de France.

Clot, Y. (2005). Le développement du collectif : entre l'individu et l'organisation du travail. In R. Teulier \& P. Lorino, Entre connaissance et organisation : l'activité collective, (pp.187-199). Paris : La Découverte.

Clot, Y. (2008). Travail et pouvoir d'agir. Paris : Presses Universitaires de France.

Clot, Y., \& Faïta, D. (2000). Genres et styles en analyse du travail. Travailler, 4, 7-42.

Clot, Y., Faïta, D., Fernandez, G. \& Scheller, L. (2001). Entretiens en autoconfrontation croisée: une méthode en clinique de l'activité. Education Permanente. Dossier Clinique de l'activité et pouvoir d'agir, 146, 17-25.

Coffey, H. (2010). "They taught me": the benefits of early community-based field experiences in teacher education. Teaching and Teacher Education, 26, 2, 335342.

Cuvelier, L., \& Caroly, S. (2009). Appropriation d'une stratégie opératoire: un enjeu du collectif de travail, @ctivités, 6, 2, 57-74.

Escalié, G., \& Chaliès, S. (2009). Optimiser le conseil pédagogique : la mise au travail d'un collectif de formation. Travail et formation en éducation [En ligne], 4 | 2009.

Escalié, G., \& Chaliès, S. (2012). Penser l'alternance des enseignants en formation à l'appui d'une communauté élargie de formateurs. In S. Ciavaldini-Cartaut (Eds.), Innover en formation : accompagner autrement les enseignants entrant dans le métier, (pp. 81-85). Paris: L'Harmattan.

Escalié, G., \& Chaliès, S. (2016). Supporting the work arrangements of cooperating teachers and university supervisors to better train preservice teachers: a new theoretical contribution. European Journal of Teacher Education, 39, 3, 302-319.

Flandin, S., \& Ria, L. (2014). Étude de l'activité d'un stagiaire d'EPS au travail et en vidéoformation autonome. Une " traçabilité » de l'évolution de l'intervention professionnelle. Recherches \& Éducations, 12, 57-73.

Gaudin, C., Perrot, F., Chaliès, S., Escalié, G., \& Raymond, J. (2015). La visite de stage : de l'observation au conseil à partir des règles de métier. Revue E.P.S, 363, 38-41.

Gervais, C. (2008). Pour une formation des stagiaires en concertation. In M. Boutet \& J. Pharand (Dir.), L'accompagnement concerté des stagiaires en enseignement, (pp. 91-107). Québec : Presses Universitaires du Québec.

MENESR la loi $n^{\circ}$ 2013-595 sur la refondation de l'école de la République du 8 juillet 2013. 


\section{eJRIEPS 41 avril 2017}

Leontiev, A.N. (1984). Activité, conscience, personnalité. Moscou : Éditions du Progrès.

Meegan, S., Dunning, C., Belton, S., \& Woods, C. (2013). Teaching practice: University supervisors' experiences and perceptions of a cooperating physical education teacher education programme. European Physical Education Review, doi: $10.1177 / 1356336 \times 13486054$.

Moussay, S. (2012). Le tutorat collectif comme ressource professionnelle au service de l'apprentissage du métier d'enseignant. In S. Ciavaldini-Cartaut (coord), Innover en formation Accompagner autrement les enseignants entrant dans le métier, (pp. 60-71). Paris : L'Harmattan.

Moussay, S., \& Serres, G. (2015). Apprendre à devenir formateur d'enseignants : vers une nouvelle professionnalité. In V. Lussi-Borer \& L. Ria (Eds.), Apprendre à enseigner, (pp. 209-220). Paris : Presses Universitaires de France.

Moussay, S. (2016). Le tutorat mixte : quels bénéfices et quels coûts ? Journée d'accueil des Tuteurs, Rectorat Académie Clermont-Ferrand, le 7 septembre 2016.

Quillerou-Grivot, E., \& Clot, Y. (2013). Trois conditions pour une clinique de l'activité en psychologie du travail : le cas d'une intervention dans une entreprise de logistique automobile. @ctivités, 10, 2, 229-248.

Rapport IGAENR 2015-044: Tutorat des futurs enseignants et conseillers principaux d'éducation, juin 2015.

Rapport IGEN-IGAENR 2015-081 : Le suivi de la mise en place des écoles supérieures du professorat et de l'éducation au cours de l'année 2014-2015, octobre 2015.

Roche, L., \& Gal-Petitfaux, N. (2014). Acquérir des gestes professionnels en EPS grâce à un dispositif de vidéo-formation. Recherches \& éducations, 131-146.

Scheller, L. (2013). Travail, affects, activité transférentielle », @ctivités, 10, 2, http://activites.revues.org/853

Van Nieuwenhoven, C., Colognesi, S. Wattiez, R., \& Bothy, E. (2016). Renforcer la dynamique collaborative entre des maitres de stage et des superviseurs par la coconstruction d'un modèle d'accompagnement. Colloque de l'ADMEE, Lisbonne du 13/01/2016 au 15/01/2016.

Vygotski, L.S. (1934/1997). Pensée et langage. Paris : La Dispute.

Wang, L., \& Ha, A. (2012). Mentoring in TGfU teaching: Mutual engagement of pre-service teachers, cooperating teachers and university supervisors. European Physical Education Review, 18, 47-6. 
eJRIEPS 41 avril 2017

Yvon, F., \& Clot, Y. (2003). Apprentissage et développement dans l'analyse du travail enseignant. Revista da Psicologia da Educação, 19, 11-38.

Yvon, F., \& Join-Lambert, R. (2007). Un cadre d'intervention à l'épreuve du réel: développer l'activité d'un groupe de formateurs d'enseignants. Education et Francophonie, XXXV, 2, 24-44. 